\title{
Consideration on TMPRSS2 and the risk of COVID-19 infection in Cushing's syndrome
}

\author{
Mattia Barbot $\mathbb{1}^{1} \cdot$ Filippo Ceccato $^{1} \cdot$ Carla Scaroni $^{1}$
}

Received: 5 May 2020 / Accepted: 10 June 2020 / Published online: 24 June 2020

(c) Springer Science+Business Media, LLC, part of Springer Nature 2020

Patients with Cushing's syndrome (CS) have an increased susceptibility to infections that contribute to their higher mortality. Cortisol excess reduces B-cell development and proliferation, $\mathrm{T}$ helper 1 lymphocyte response, and induces neutrophilia with an increase in neutrophil/lymphocyte (N/L) ratio in a concentration-dependent manner [1]. These changes are associated with more severe, protracted clinical course, and disseminated forms of common viral infections such as influenza and likely also to the novel severe acute respiratory syndrome coronavirus 2 (SARS-CoV-2). Given the high mortality of untreated CS, a rapid surgical approach to the cause of the hormonal excess is the first-tier option. In the current pandemic scenario, access to surgical procedure have been necessarily restrained, as most of our affords have been shifted to handle COVID-19 patients. Moreover, hospitalization and surgical stress are associated with changes in immune profile that can further exacerbate immunodepression in CS patients. Alternative therapies based on cortisol-lowering medications, usually considered second-line option after surgical failure, can actually allow to reschedule the intervention. Commercially available steroidogenesis inhibitors, such as ketoconazole and metyrapone, have several advantages due to the convenient way of administration, rapid onset of action and their possible use in all type of CS. Furthermore, they act on androgens secretion which has recently become of interest in SARS-COV-2 infection; androgen action has been pointed out as a potential contributor to the higher prevalence of COVID-19 among men and their worse outcome compared to female [2]. A possible explanation to this gender-related difference is connected to the expression of

Mattia Barbot

mattiabarbot@alice.it

1 Endocrinology Unit, Department of Medicine DIMED, University-Hospital of Padova, Padova, Italy transmembrane protease serine 2 (TMPRSS2) in bronchial epithelium whose expression is finely regulated by androgen signaling. TMPRSS2, firstly studied in prostate cancer, was indeed found as essential to activate the spikes, induce viral spread, and pathogenesis in the infected hosts [3].

Since CS is a condition frequently associated with androgen excess in female, it is a fair assumption to speculate that these patients might have higher expression of TMPRSS2 and consequently an increased risk of COVID19 infection. Cortisol excess can be easily reverted by effective surgery; however, given the restricted access to surgical operation theater, interventions are not feasible at this very moment. Not to mention that CS carries important comorbidities such as hypertension, diabetes, and increase venous thromboembolism, all of which have already been associated with poorer prognosis in infected patients [1].

Lastly, the fact that both SARS-Cov-2 infection and CS itself contribute to increase N/L ratio, in turn associated with more severe disease, questions the legitimacy to operate these patients in such setting.

Bearing this in mind, CS patients should probably be temporary managed with medical therapy, choosing ketoconazole as first medical option due to its effect of inhibition of androgens secretion. Ketoconazole is an imidazole derivative antifungal agent that inhibits multiple cytochrome P450 enzymes, namely side-chain cleavage, 17,20lyase, 11-b-hydroxylase, and 17-a-hydroxylase. The overall result in both genders is an antiandrogen action. Ketoconazole is generally well tolerated with mild gastrointestinal side effects, and has an overall efficacy around $50 \%$ with concomitant improvement of signs and symptoms. Severe hepatitis is very rare, but liver function monitoring is necessary on a monthly basis for 3 months, as well as at therapy initiation or at dose increase. When a CS patient is already affected by COVID-19 infection, it is probably more careful to withdraw ketoconazole therapy due to potential drug-drug interactions and possible QTc interval elongation. To add, androgen blockade becomes questionable once symptoms are already present, because 
TMPRSS2 seems to be involved in the early phase of infection.

Even though metyrapone treatment has a quicker onset of action than ketoconazole, it may cause the accumulation of androgenic precursors secondary to the blockade of cortisol synthesis, that can virtually enhance TMPRSS2 expression and favor infection.

In conclusion, there is a need to adapt our usual endocrine care-path to the pandemic crisis. Cortisol-lowering medication with concomitant antiandrogen therapy might prove beneficial on viral load and severity of lung involvement. Although the effectiveness of antiandrogen therapy is still purely speculative, it is worth a try; we therefore suggest to prefer ketoconazole over other medications to reduce host vulnerability to COVID-19 in newly diagnosed CS. Until the contribution of androgen will be clarified, there is no need to shift patients already on treatment with other cortisol-lowering medications to ketoconazole, if they have satisfactory hormonal control. Surgery remains the only curative option and should be performed as soon as clinical activity returns to normal. Further studies are still required to verify this hypothesis and confirm the role of antiandrogen therapy in COVID-19 prevention.

\section{Compliance with ethical standards}

Conflict of interest The authors declare that they have no conflict of interest.

Publisher's note Springer Nature remains neutral with regard to jurisdictional claims in published maps and institutional affiliations.

\section{References}

1. R. Pivonello, A.M. Isidori, M.C. De Martino, J. Newell-Price, B.M. Biller, A. Colao, Complications of Cushing's syndrome: state of the art. Lancet Diabetes Endocrinol. 4(7), 611-629 (2016). https://doi. org/10.1016/S2213-8587(16)00086-3

2. G. Grasselli, A. Zangrillo, A. Zanella, M. Antonelli, L. Cabrini, A. Castelli, D. Cereda, A. Coluccello, G. Foti, R. Fumagalli, G. Iotti, N. Latronico, L. Lorini, S. Merler, G. Natalini, A. Piatti, M.V. Ranieri, A.M. Scandroglio, E. Storti, M. Cecconi, A. Pesenti, COVID-19 Lombardy ICU Network. Baseline Characteristics and Outcomes of 1591 Patients Infected With SARS-CoV-2 Admitted to ICUs of the Lombardy Region, Italy. JAMA. 2020. https://doi. org/10.1001/jama.2020.5394

3. K.H. Stopsack, L.A. Mucci, E.S. Antonarakis, P.S. Nelson, P.W. Kantoff, TMPRSS2 and COVID-19: Serendipity or Opportunity for Intervention? Cancer Discov. 2020. https://doi.org/10.1158/21598290.CD-20-0451 